\title{
Catchments as space-time filters - a joint spatio-temporal geostatistical analysis of runoff and precipitation
}

\author{
J. O. Skøien and G. Blöschl \\ Institute for Hydraulic and Water Resources Engineering, Vienna Univ. of Technology, Karlsplatz 13, 1040 Vienna, Austria
}

Received: 29 March 2006 - Published in Hydrol. Earth Syst. Sci. Discuss.: 12 June 2006

Revised: 30 August 2006 - Accepted: 18 September 2006 - Published: 26 September 2006

\begin{abstract}
In this paper catchments are conceptualised as linear space-time filters. Catchment area $A$ is interpreted as the spatial support and the catchment response time $T$ is interpreted as the temporal support of the runoff measurements. These two supports are related by $T \sim A^{\kappa}$ which embodies the space-time connections of the rainfall-runoff process from a geostatistical perspective. To test the framework, spatiotemporal variograms are estimated from about 30 years of quarter hourly precipitation and runoff data from about 500 catchments in Austria. In a first step, spatio-temporal variogram models are fitted to the sample variograms for three catchment size classes independently. In a second step, variograms are fitted to all three catchment size classes jointly by estimating the parameters of a point/instantaneous spatiotemporal variogram model and aggregating (regularising) it to the spatial and temporal scales of the catchments. The exponential, Cressie-Huang and product-sum variogram models give good fits to the sample variograms of runoff with dimensionless errors ranging from 0.02 to 0.03 , and the model parameters are plausible. This indicates that the first order effects of the spatio-temporal variability of runoff are indeed captured by conceptualising catchments as linear space-time filters. The scaling exponent $\kappa$ is found to vary between 0.3 and 0.4 for different variogram models.
\end{abstract}

\section{Introduction}

Geostatistical methods fall into two groups. The first focuses on the characterisation of spatial variability and is termed structural analysis. It provides a representation of the spatial structure of the variables of interest in terms of the variogram and sheds light on the continuity of the processes involved. In hydrology, structural analysis plays an impor-

Correspondence to: J. O. Skøien

(skoien@hydro.tuwien.ac.at) tant role in aquifer assessment and sampling design (e.g., James and Freeze, 1993). The second group of geostatistical methods consists of spatial estimation methods where the variogram obtained in the structural analysis step is used to estimate the variable of interest at locations where no measurements are available. Spatial estimation methods based on geostatistical concepts are widely used in many geosciences including subsurface hydrology (Renard et al., 2005).

In catchment hydrology, geostatistical concepts have been used more sparingly. This is because of the nested structure of catchments which makes geostatistical analyses more complicated as compared to the usual analysis of point samples or blocks. However, a number of recent studies have demonstrated that geostatistical methods can indeed account for the nested catchment structure. This applies to both the structural analysis step of understanding the spatial structure and the spatial estimation step of estimating variables such as streamflow at locations where no data are available. The latter addresses the ungauged catchment problem (Sivapalan et al., 2003). Based on the work of Gottschalk (1993a, b); Sauquet et al. (2000) presented a spatial estimation method for annual streamflow. A similar spatial estimation method, termed TOPKRIGING, was presented by Skøien et al. (2005) who showed that accounting for the nested catchment structure improved the spatial estimates of flood frequency over a method that did not account for nested catchments. Reliable variograms are needed for applying this type of spatial estimation methods.

Runoff is a process that varies in both space and time. It is therefore appealing to extend the spatial analyses of Sauquet et al. (2000) and Skøien et al. (2005) to the spatiotemporal case, i.e. to analyse and estimate runoff as a function of both space and time. Spatio-temporal variograms are needed for this. At the same time, spatio-temporal variograms of runoff may shed light on the nature of hydrological variability in space and time. Skøien et al. (2003) analysed the effect of different catchment sizes on the spatial and

Published by Copernicus GmbH on behalf of the European Geosciences Union. 

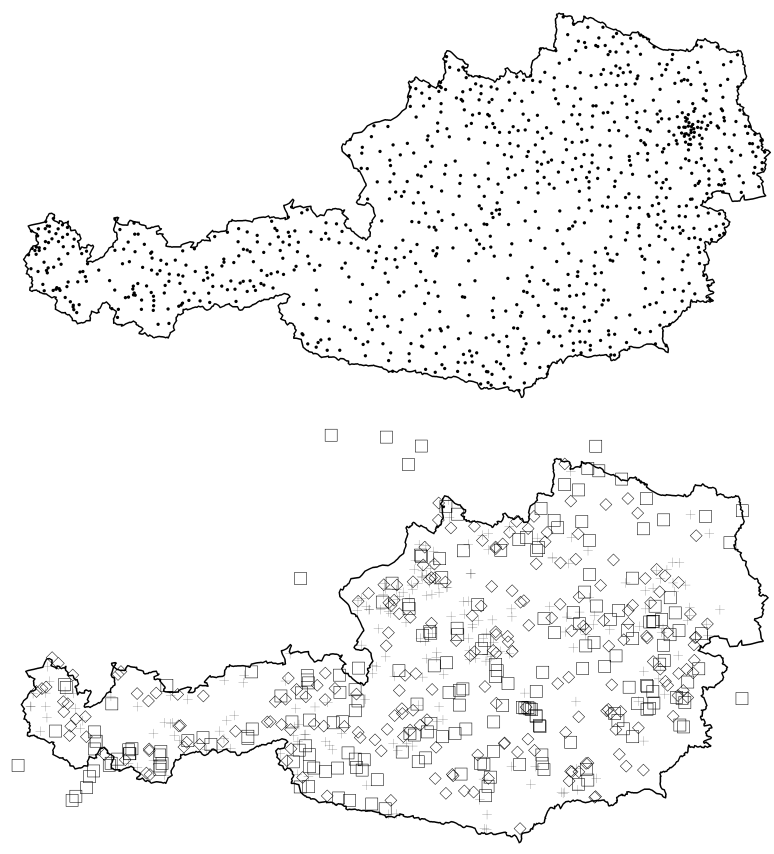

Fig. 1. Network of measurement stations in Austria used in this paper. Precipitation gauges (top); centroids of gauged catchments (bottom) (small catchments shown as plusses, medium sized catchments as diamonds, large catchments as squares).

temporal variograms of precipitation and runoff. Their results indicated that variograms of observed runoff were consistent with variograms obtained by aggregating variograms of hypothetical point runoff. However, their study examined spatial and temporal variograms independently. It is likely that the spatial and temporal variabilities of runoff are related given that it takes longer for water to move through large catchments than through small catchments. Woods et al. (1995) analysed catchments in the range of $0.04-50 \mathrm{~km}^{2}$ and found the variance of streamflow to decrease more strongly with catchment area than what would be expected for the spatial aggregation of a random process. Woods et al. (1995) noted that this may be due to the presence of organisation at large scales that is not present at small scales but Skøien et al. (2003) suggested that this may be related to spatio-temporal aggregation effects instead.

It is therefore time to follow the suggestion of Skøien et al. (2003) to analyse runoff in space and time jointly, and to examine the joint spatial and temporal aggregation effects of runoff. As a central concept, we adopt the description of Woods and Sivapalan (1999), where runoff from a catchment is represented as the convolution of the local runoff generated within the catchment within a time period. This is consistent with the filter concept of Skøien et al. (2003) where the catchment area is interpreted as the geostatistical support of the runoff measurements. In a joint spatio-temporal analysis the catchments then operate as space-time filters and runoff measurements are associated with both a spatial support (the catchment area) and a temporal support (the response time of the catchment).

The objective of this paper is to analyse spatio-temporal variograms of runoff and examine the potential of estimating these from point variograms of runoff by spatio-temporal aggregation. For comparison and for testing the aggregation procedure, spatio-temporal variograms of precipitation are analysed as well. This study uses a similar data set as Skøien et al. (2003) but goes beyond their study in two ways. First, Skøien et al. (2003) analysed the variograms in space and time separately while, here, a joint analysis is performed to shed light on the connection of space and time scale variability. Second, Skøien et al. (2003) used a data set of daily values while, here, a data set of quarter hourly values is used. This allows us to perform a more detailed analysis of the short term characteristics of runoff that are important for space-time connections.

\section{Data}

The data used in this paper stem from a comprehensive hydrographic data set of Austria. Austria has a varied climate with mean annual precipitation ranging from $500 \mathrm{~mm}$ in the eastern lowland regions up to about $3000 \mathrm{~mm}$ in the western alpine regions. Runoff depths range from less than $50 \mathrm{~mm}$ per year in the eastern part of the country to about $2000 \mathrm{~mm}$ per year in the Alps. Potential evapotranspiration is on the order of 600-900 mm per year. Precipitation data from 991 stations for the period 1981-1997 were used in this study (Fig. 1a). 161 of the stations were recording rain gauges while the rest were daily raingauges. The daily records were disaggregated to a time step of $15 \mathrm{~min}$ based on the temporal patterns of the neighbouring stations (Merz et al., 2006). In order to be able to examine spatial aggregation effects, catchment precipitation was calculated for each time step by external drift kriging interpolation of the point data for a total of 579 catchments using topographic elevation as an auxiliary variable. The catchment precipitation series so obtained were divided into three size classes (Table 1). Runoff data from 591 catchments for the period 1971-2000 were used that all had a time resolution of $15 \mathrm{~min}$. The catchments were subdivided into three classes according to catchment size small $\left(3-71 \mathrm{~km}^{2}\right)$, medium $\left(72-250 \mathrm{~km}^{2}\right)$ and large $(250-$ $131000 \mathrm{~km}^{2}$ ) (Fig. 1b). Catchments smaller than $10 \mathrm{~km}^{2}$, as well as catchments with short records, significant anthropogenic effects or lake effects were excluded from the data set. This resulted in a total of 488 stream gauges available for the analysis. Table 1 summarises the data series used in this paper. The runoff data set consists of a total of $5 \times 10^{8}$ individual data values. 
Table 1. Data series used in this paper.

\begin{tabular}{|c|c|c|c|c|c|c|c|}
\hline Data type & Size class & $\begin{array}{l}\text { Size range } \\
\left(\mathrm{km}^{2}\right)\end{array}$ & $\begin{array}{l}\text { Median size } \\
\left(\mathrm{km}^{2}\right)\end{array}$ & $\begin{array}{l}\text { Number of } \\
\text { stations }\end{array}$ & $\begin{array}{c}\text { Extent of domain } \\
(\mathrm{km})\end{array}$ & $\begin{array}{l}\text { Time resolution } \\
\text { (min) }\end{array}$ & Period \\
\hline Point precipitation & - & Point & Point & 991 & 700 & 15 & 1981-1997 \\
\hline \multirow[t]{3}{*}{ Catchment precipitation } & Small & $3-71$ & 35 & 193 & 700 & 15 & 1981-1997 \\
\hline & Medium & $72-236$ & 125 & 193 & 700 & 15 & 1981-1997 \\
\hline & Large & $241-131000$ & 670 & 193 & 700 & 15 & 1981-1997 \\
\hline \multirow[t]{3}{*}{ Runoff } & Small & $10-71$ & 42 & 142 & 700 & 15 & $1971-2000$ \\
\hline & Medium & $72-248$ & 119 & 178 & 700 & 15 & $1971-2000$ \\
\hline & Large & $251-131000$ & 605 & 168 & 700 & 15 & $1971-2000$ \\
\hline
\end{tabular}

\section{Method}

\subsection{Spatio-temporal sample variograms}

Spatio-temporal sample variograms were calculated from the runoff data separately for the three catchment size classes, and from catchment precipitation separately for the three catchment size classes as well as for point precipitation:

$$
\begin{aligned}
& \hat{\gamma}_{s t}\left(h_{s}, h_{t}\right)=\frac{1}{2 \sum_{j=1}^{m\left(h_{s}\right)} n_{j}\left(h_{t}\right)} \\
& \sum_{j=1}^{m\left(h_{s}\right)} \sum_{i=1}^{n_{j}\left(h_{t}\right)}\left(z\left(\boldsymbol{x}_{\boldsymbol{j}}+\boldsymbol{h}_{\boldsymbol{s}}, t_{i}+h_{t}\right)-z\left(\boldsymbol{x}_{\boldsymbol{j}}, t_{i}\right)\right)^{2}
\end{aligned}
$$

where $h_{s}=\left|\boldsymbol{h}_{\boldsymbol{s}}\right|$ and $h_{t}$ are the spatial and temporal lags, respectively, $z\left(\boldsymbol{x}_{\boldsymbol{j}}, t_{i}\right)$ is precipitation or runoff at time $t_{i}$ and spatial location $\boldsymbol{x}_{\boldsymbol{j}}$ of station $j, m\left(h_{s}\right)$ is the number of pairs of stations with distance $h_{s}$, and $n_{j}\left(h_{t}\right)$ is the number of pairs of points in time with time lag $h_{t}$ within a spatial or temporal bin. $h_{s}$ was taken as the distance between the centres of gravity of the catchments for the cases of runoff and catchment precipitation and as the station distance for the case of point precipitation. The spacings of the bins were selected approximately logarithmically (with the exception of zero lags). The variograms of precipitation were calculated on the basis of precipitation intensity, those of runoff on the basis of specific discharge. The physical units of the precipitation and runoff variograms hence are $\left(\mathrm{mm}^{2} \times \mathrm{h}^{-2}\right)$ and $\left(\mathrm{m}^{6} \times \mathrm{km}^{-4} \times \mathrm{s}^{-2}\right)$ with $1 \mathrm{~m}^{6} \times \mathrm{km}^{-4} \times \mathrm{s}^{-2}=12.96 \mathrm{~mm}^{2} \times \mathrm{h}^{-2}$. The space and time units used are kilometres and hours, respectively.

\subsection{Spatio-temporal variogram models}

Numerous spatio-temporal variogram models have been proposed in the literature. There are two types, separable and non-separable models. In separable models, the covariance can be factorised into two components, one component containing time lag only and the other containing space lag only.
Rodríguez-Iturbe and Mejía (1974) presented an example of a separable model. Cressie and Huang (1999) proposed a series of non-separable models. De Cesare et al. (2001) and De Iaco et al. (2001) extended some of the earlier models into a product-sum model. Kyriakidis and Journel (1999) reviewed spatio-temporal variogram models and discussed advantages and disadvantages of different model types. Fuentes (2006) and Mitchell et al. (2005) proposed methods for testing if a process can be modelled by a separable model. They noted that for some spatio-temporal modelling applications, the computational burden can be reduced considerably by using separable models. Cressie and Huang (1999), however, suggested that non-separable models are necessary for many natural cases.

Four models are compared in this paper that are all nonseparable: a spatio-temporal exponential model, a model proposed by Cressie and Huang (1999), the product-sum model (De Cesare et al., 2001; De Iaco et al., 2001), to all of which a fractal component was added (Eq. 8), as well as a pure fractal model. The exponential model is:

$\gamma_{1 s t}^{\prime}\left(h_{s}, h_{t}\right)=a_{1}\left(1-\exp \left(-\left(\left(c_{1} h_{t}+h_{s}\right) / d_{1}\right)^{e_{1}}\right)\right)$

$a_{1}$ is the sill or the variance for infinite lag, $c_{1}$ is a scaling parameter for time, $d_{1}$ is a spatio-temporal correlation length and $e_{1}$ defines the slope of the short distance part of the variogram. The model is consistent with the Taylor hypothesis which assumes that a constant characteristic velocity exists, so space and time are interchangeable (Taylor, 1938; Skøien et al., 2003). Cressie and Huang (1999) derived a number of models from Bochner's theorem (Bochner, 1955). We tested a number of them and focus in this paper on:

$\gamma_{2 s t}^{\prime}\left(h_{s}, h_{t}\right)=a_{2}\left(1-\frac{1}{\left(c_{2} h_{t}+1\right)^{(d+1) / 2}} \exp \left\{-\frac{b_{2}^{2} h_{s}^{2}}{c_{2} h_{t}+1}\right\}\right)$

$a_{2}$ is the sill, $b_{2}$ and $c_{2}$ are scaling parameters for space and time, respectively, and $d$ is the spatial dimension.

The third model is the product-sum model which is derived from a covariance model that combines products and sums (De Cesare et al., 2001; De Iaco et al., 2001):

$\gamma_{3 s t}^{\prime}\left(h_{s}, h_{t}\right)=\gamma_{3 s}^{\prime}\left(h_{s}\right)+\gamma_{3 t}^{\prime}\left(h_{t}\right)-k \gamma_{3 s}^{\prime}\left(h_{s}\right) \gamma_{3 t}^{\prime}\left(h_{t}\right)$ 
where $k$ is a parameter. $\gamma_{3 s}^{\prime}\left(h_{s}\right)$ and $\gamma_{3 t}^{\prime}\left(h_{t}\right)$ represent the spatial and temporal variograms, respectively:

$\gamma_{3 s}^{\prime}\left(h_{s}\right)=a_{3 s}\left(1-\exp \left(-\left(h_{s} / d_{3 s}\right)^{e_{3 s}}\right)\right)$

$\gamma_{3 t}^{\prime}\left(h_{t}\right)=a_{3 t}\left(1-\exp \left(-\left(h_{t} / d_{3 t}\right)^{e_{3 t}}\right)\right)$

with parameters similar to Eq. (2). The product-sum model reduces to the separable model proposed by RodríguezIturbe and Mejía (1974) for $\alpha_{3 s}=\alpha_{3 t}=1 / k$. The three variogram models (Eqs. 2, 3, 4) are stationary, i.e., they are finite for infinite lags. Skøien et al. (2003) showed that daily precipitation can be regarded as stationary in time, daily mean runoff is almost stationary in time, while neither of the processes can be regarded as stationary in space within the spatial extent of the data set used. The variograms were therefore modified to account for non-stationarity in both spatial and temporal directions. Although Skøien et al. (2003) noted that runoff was almost stationary in time, a small nonstationary part was found to be necessary for the regularisation procedure in this paper. For application in spatial (and spatio-temporal) estimation a variogram needs to be such that the variance of any linear combination $Y$ of the variable $z$ of the type $Y=\sum_{i=1}^{n} \alpha_{i} z\left(\boldsymbol{x}_{\boldsymbol{i}}, t_{i}\right)$ is equal to zero or positive. This requirement is fullfilled by Eqs. $(2,3,4)$. If the variogram is non-stationary, the following condition has to be fulfilled:

$\operatorname{Var}(Y)=-\sum_{i} \sum_{j} a_{i} a_{j} \gamma_{s t}\left(\left|\boldsymbol{x}_{\boldsymbol{i}}-\boldsymbol{x}_{\boldsymbol{j}}\right|, t_{i}-t_{j}\right) \geq 0$

with $\sum_{i=1}^{n} \alpha_{i}=0 .-\gamma_{s t}\left(h_{s}, h_{t}\right)$ is then by definition said to be a "conditional positive definite function" (Journel and Huijbregts, 1978; Cressie, 1991). To ensure conditional positive definiteness of $-\gamma_{s t}\left(h_{s}, h_{t}\right)$, it is common to specify the variogram as a sum or a product of models that are known to have this property. We have therefore added spatial and temporal fractal components that are positive definite to the three variogram models $\gamma^{\prime}{ }_{s t}$ of Eqs. $(2,3,4)$ :

$\gamma_{s t}=\gamma_{s t}^{\prime}+a_{s} h_{s}^{\alpha}+a_{t} h_{t}^{\beta}$

where $a_{s}$ and $a_{t}$ are parameters that adjust the level of the fractal part, and $\alpha$ and $\beta$ are the spatial and temporal fractalities, $0<\alpha<2$ and $0<\beta<2$. Although this model ensures conditional positive definiteness, the non-stationary part (Eq. 8) does not include space-time interactions. In addition to the three variogram models, we examined a pure fractal model for comparison (Eq. 12 below). In summary, the following variogram models were used in this paper:

Exponential model:

$$
\begin{aligned}
& \gamma_{1 s t}\left(h_{s}, h_{t}\right)= \\
& a_{1}\left(1-\exp \left(-\left(\left(c_{1} h_{t}+h_{s}\right) / d_{1}\right)^{e_{1}}\right)\right)+a_{s} h_{s}^{\alpha}+a_{t} h_{t}^{\beta}
\end{aligned}
$$

Cressie-Huang model:

$$
\begin{gathered}
\gamma_{2 s t}\left(h_{s}, h_{t}\right)=a_{2}\left(1-\frac{1}{\left(c_{2} h_{t}+1\right)^{(d+1) / 2}} \exp \left\{-\frac{b_{2}^{2} h_{s}^{2}}{c_{2} h_{t}+1}\right\}\right) \\
+a_{s} h_{s}^{\alpha}+a_{t} h_{t}^{\beta}
\end{gathered}
$$

Product-sum model (using Eqs. 5 and 6):

$$
\begin{aligned}
& \gamma_{3 s t}\left(h_{s}, h_{t}\right)= \\
& \gamma_{3 s}^{\prime}\left(h_{s}\right)+\gamma_{3 t}^{\prime}\left(h_{t}\right)-k \gamma_{3 s}^{\prime}\left(h_{s}\right) \gamma_{3 t}^{\prime}\left(h_{t}\right)+a_{s} h_{s}^{\alpha}+a_{t} h_{t}^{\beta}
\end{aligned}
$$

Fractal model:

$\gamma_{4 s t}=a_{s} h_{s}^{\alpha}+a_{t} h_{t}^{\beta}$

\subsection{Spatio-temporal regularisation}

\subsubsection{Concept of catchments as space-time filters}

Measurements are strongly affected by the measurement scale. Blöschl and Sivapalan (1995) formulated the measurement scale as a scale triplet: the distance between measurements (spacing); the size of the region over which measurements are available (extent); and the area or volume that each measurement represents (support). Skøien and Blöschl (2006a) and Skøien and Blöschl (2006b) performed coherent studies of measurement scale effects on parametric and non-parametric estimates of spatial correlation, respectively. As the support increases, the variable of interest becomes increasingly smoother. Because of this, the variance (and hence the sill of the variogram) decreases and the correlation lengths increase.

In this paper, we interpret the catchment area as the spatial support of the runoff measurements and conceptualise local runoff as a point process following Woods and Sivapalan (1999) and Skøien et al. (2005). In a joint spatio-temporal analyses both the spatial and the temporal supports need to be taken into account. In this paper, we therefore interpret the response time of a catchment as the temporal support. Runoff at the catchment outlet is then assumed to be some sort of aggregated value of local runoff over the catchment area (spatial support) over the catchment response time (temporal support).

The concept starts with local runoff or rainfall excess, $R(x$, $y, t)$. To account for routing on the hillslopes and in the channels within the catchment, a weighting function $u(x, y$, $t$ ) is introduced which allows to combine local instantaneous runoff into runoff at the catchment outlet, $Q_{i}$ :

$$
Q_{i}(t)=\iint_{A_{i}} \int_{t-T_{i}}^{t} R(x, y, \tau) u(x, y, \tau) d \tau d x d y
$$

where $A_{i}$ is the area of catchment $i, T_{i}$ is the time interval that influences the output, $x$ and $y$ are the space coordinates, $t$ is time and $\tau$ is the temporal integration variable. The weighting function $u(x, y, t)$ represents the routing processes within 
the catchment and varies in space. For example, runoff generated close to the outlet or close to the streams will reach the outlet faster than runoff generated further away. Also, $u(x$, $y, t)$ will be a function of catchment characteristics such as hill slope orientation, catchment slope and soil types. $u(x$, $y, t$ ), for a certain point in space, also changes with time as the flow velocities change with changes in the catchment state. As an approximation, we assume in this paper that, for a given catchment, the weighting function is constant within the integration limits both in space and time, i.e., $u_{i}=1 / T_{i}$. For a constant weighting function, Eq. (13) becomes a linear filter or a convolution integral. In time, the weighting function is equivalent to a unit hydrograph that is constant between 0 and $T_{i}$ and zero elsewhere. In space, the weighting function is constant within the catchment area and zero elsewhere which is consistent with the assumptions of Sauquet et al. (2000) and Skøien et al. (2003). The specific runoff at the catchment outlet (runoff divided by catchment area) then becomes:

$q_{i}(t)=\frac{1}{A_{i} T_{i}} \iint_{A_{i}} \int_{t-T_{i}}^{t} R(x, y, t-\tau) d \tau d x d y$

The runoff routing process is hence conceptualised as a linear space-time filter in this paper. For simplicity, we assume that the filter kernel in space is a square with area $A_{i}$ (catchment size), and in time the filter kernel is a block unit hydrograph with time base $T_{i}$ as mentioned above. We assume a simple relationship between catchment response time and catchment area:

$T_{i}=\mu A_{i}^{\kappa}$

where $\mu$ and $\kappa$ are parameters to be estimated from the data. For $\kappa>0$ the response time increases with catchment size. Eq. (15) embodies the space-time connections of the rainfallrunoff process from a linear filter perspective. Note that Eq. (15) applies to runoff. For comparison, we also analysed catchment precipitation for which we used the same aggregation procedure in space but a constant temporal support of $T_{i}=15 \mathrm{~min}$, as consistent with the raingauge data.

In a geostatistical framework, the linear aggregation of Eq. (14) is represented by the second moments. A point variogram of runoff represents the second moment of lo$\mathrm{cal}$, instantaneous runoff. From the point variogram with zero support in space and zero support in time (i.e. instantaneous) one can estimate variograms that are valid for finite support areas and finite support times by a procedure that is usually referred to as regularisation (Journel and Huijbregts, 1978). Conversely, it is possible to back-calculate the point/instantaneous variogram from variograms based on finite supports (Skøien et al., 2003). The point variograms are the basis of spatial estimation methods such as those of Sauquet et al. (2000) and Skøien et al. (2005). In addition, the point variogram sheds light on the spatio-temporal structure of instantaneous runoff generated at the local scale.

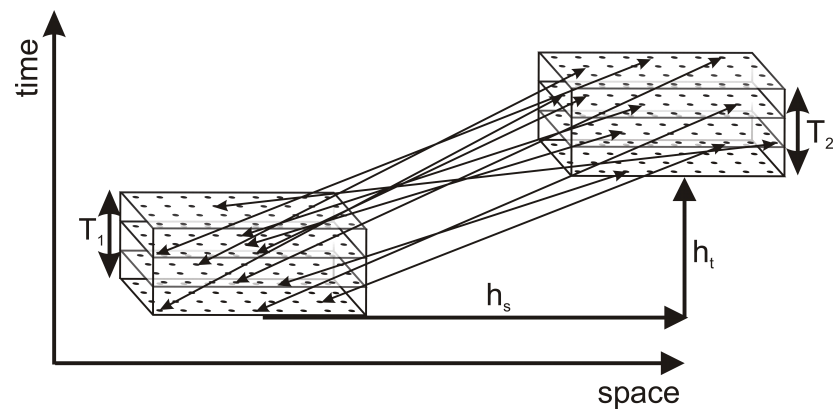

Fig. 2. Schematic of variance estimation between two catchments 1 and 2 and a range of time lags. Thin arrows represent some of the spatio-temporal pairs of data points.

\subsubsection{Implementation}

The variogram value, given a certain distance, represents the expected variance of a process within an extent equal to this distance. If a variable is linearly aggregated, each measurement is the average of the point process within the support of the measurement. If we assume that the variance of catchment runoff is both dependent on the spatial and temporal supports ( $A$ and $T$, respectively, dropping the index), for two catchments of equal size the spatial regularisation technique of (Cressie, 1991, 66) can be extended to:

$$
\begin{aligned}
& \gamma_{s t}\left(h_{s}\left|a, h_{t}\right| T\right)= \\
& \frac{1}{A^{2} T^{2}} \int_{A} \int_{A} \int_{T} \int_{T} \gamma_{s t}\left(\left|\boldsymbol{r}_{\mathbf{1}}+\boldsymbol{h}_{\boldsymbol{s}}-\boldsymbol{r}_{\mathbf{2}}\right|,\left|\tau_{1}+h_{t}-\tau_{2}\right|\right) d \boldsymbol{r}_{\mathbf{1}} d \boldsymbol{r}_{\mathbf{2}} d \tau_{1} d \tau_{2}- \\
& \frac{1}{A^{2} T^{2}} \int_{A} \int_{A} \int_{T} \int_{T} \gamma_{s t}\left(\left|\boldsymbol{r}_{\mathbf{1}}-\boldsymbol{r}_{\mathbf{2}}\right|,\left|\tau_{1}-\tau_{2}\right|\right) d \boldsymbol{r}_{\mathbf{1}} d \boldsymbol{r}_{\mathbf{2}} d \tau_{1} d \tau_{2}
\end{aligned}
$$

where $\gamma_{s t}(r, \tau)$ is the spatio-temporal variogram of the instantaneous point process, $\boldsymbol{h}_{\boldsymbol{s}}$ is the separation vector between two catchments (with space lag $h_{s}=\left|\boldsymbol{h}_{s}\right|$ ), $h_{t}$ is the time lag and $a$ is the side length of the square that approximates a catchment, i.e., $a=\sqrt{A}$. The catchment size $A$ has been taken as the median catchment size for all catchments of a given size class (Table 1). Eq. 16 indicates that the regularised variogram value between two catchments of size $A$ with response time $T$ is the variance integrated in time and space between the two catchments, minus the integrated variance within one catchment. This concept is illustrated in Fig. 2. Each catchment is visualised as a spatio-temporal "volume" separated by spatio-temporal distances.

The number of integrals has been reduced here by using the distribution function of spatio-temporal distances within and between catchments in a similar way as Western and Blöschl (1999) and Skøien et al. (2003) but extended to space and time: 
$\gamma\left(h_{s}\left|a, h_{t}\right| T\right)=\int_{h_{t}-T}^{h_{t}+T} \int_{0}^{R \max } \gamma_{s t}(r, \tau) f_{2 s t}\left(r\left|\left(h_{s}, a\right), \tau\right|\left(h_{t}, T\right)\right) d \tau d r-$

$\int_{-T}^{T} \int_{0}^{R \max } \gamma_{s t}(r, \tau) f_{1 s t}\left(r\left|\left(h_{s}, a\right), \tau\right|\left(h_{t}, T\right)\right) d \tau d r$

$f_{1 s t}(r|a, \tau| T)$ is the probability density function (pdf) of distances in space and time within a catchment with spatial support $a$ and temporal support $T$. $f_{2 s t}\left(r\left|\left(h_{s}, a\right), \tau\right|\left(h_{t}, T\right)\right)$ is the pdf of distances in space and time between points in two catchments with a centre-tocentre distance $h_{s}$ in space and $h_{t}$ in time. $R_{\max }$ is a practical integration limit. We can assume the distances in space and time to be independent, so the $f_{1 s t}$ and $f_{2 s t}$ can be separated into spatial and temporal parts:

$$
\begin{aligned}
& \gamma_{s t}\left(h_{s}\left|a, h_{t}\right| T\right)=\int_{h_{t}-T}^{h_{t}+T} \int_{0}^{R \max } \gamma_{s t}(r, \tau) f_{2 s}\left(r \mid\left(h_{s}, a\right)\right) f_{2 t}\left(\tau \mid\left(h_{t}, T\right)\right) d \tau d r \\
& \int_{-T}^{T} \int_{0}^{R \max } \gamma_{s t}(r, \tau) f_{1 s}(r \mid a) f_{1 t}(\tau \mid T) d \tau d r
\end{aligned}
$$

$f_{1 s}$ and $f_{2 s}$ are the pdfs in space which have been evaluated as in Western and Blöschl (1999) and Skøien and Blöschl (2006ab). $f_{1 t}$ and $f_{2 t}$ are the pdfs of the temporal distances, within and between catchments, respectively, which for a block unit hydrograph are:

$$
f_{1 t}(\tau \mid T)= \begin{cases}\frac{1}{T}\left(1-\frac{\tau}{T}\right) & \tau>0 \\ \frac{1}{T}\left(1+\frac{\tau}{T}\right) & \tau \leq 0\end{cases}
$$

and:

$$
\begin{aligned}
& f_{2 t}\left(\tau \mid\left(h_{t}, T\right)\right)= \\
& \left\{\begin{array}{cc}
\left(1-\frac{h_{t}}{T}+\frac{\tau}{T}\right) /\left(h_{t}-\frac{h_{t}^{2}}{22}+\frac{T}{2}\right) & 0 \leq \tau<h_{t}, 0<h_{t}<T \\
\left(1+\frac{h_{t}}{T}-\frac{\tau}{T}\right) /\left(h_{t}-\frac{h_{t}^{2}}{2 T}+\frac{T}{2}\right) & h_{t} \leq \tau<h_{t}+T, 0<h_{t}<T \\
\frac{T-h_{t}+\tau}{T^{2}-\tau} & h_{t}-T \leq \tau<h_{t}, h_{t} \geq T \\
\frac{T+h_{t}-\tau}{T^{2}} & h_{t} \leq \tau \leq h_{t}+T, h_{t} \geq T
\end{array}\right.
\end{aligned}
$$

\subsection{Parameter estimation of variograms}

The analyses are organised into two parts. In the first part, variogram models are fitted to the sample variograms of the small, medium and large catchment size classes independently (Sects. 4.1 and 4.2). In the second part, one point variogram model is fitted jointly to the three catchment size classes based on regularisation (Sects. 4.3 and 4.4).

In the first part we used a version of the weighted leastsquares (WLS) method (Cressie, 1985) to estimate the parameters of the variogram models by minimizing the objective function:

$$
\Phi=\frac{1}{\sum_{i=1}^{n s} \sum_{j=1}^{n t} w(i, j)} \sum_{i=1}^{n s} \sum_{j=1}^{n t} w(i, j) \cdot\left[\frac{\hat{\gamma}_{s t}\left(h_{s i}, h_{t j}\right)}{\gamma_{s t}\left(h_{s i}, h_{t j}\right)}-1\right]^{2}(21)
$$

where $\hat{\gamma}_{s t}\left(h_{s i}, h_{t j}\right)$ is the sample variogram for one of the three catchment size classes or that of point rainfall (Eq. 1), $\gamma_{s t}\left(h_{s i}, h_{t j}\right)$ is one of the variogram models (Eqs. 9-12), $h_{s i}$ and $h_{t j}$ are the spatial and temporal lags, and $n s$ and $n t$ are the number of bins in space and time. $w(i, j)$ is the weight of each bin, with the indices $i$ and $j$ in spatial and temporal directions, respectively. We used the square root of the number of pairs in each bin as the weight, except that we increased this weight by a factor of 10 for $h_{s i}=0$ and $h_{t j}=0$. These lags represent the marginal variograms in space and time. In a spatio-temporal estimation procedure, the marginal variograms will be important. As the bins on the margins only constitute approximately one tenth of the total number of bins in the spatio-temporal variograms, the increased weights balance the importance of the margins with the rest of the variogram. The SCEUA-method (Duan et al., 1992) was used to search for the best parameter set. The search was carried out ten times for each model type and catchment size class with different starting values, to reduce the probability of finding local minima. The variogram models associated with the smallest objective function of the ten trials are shown. The procedure was repeated for each catchment size class (including point precipitation), each variogram model and for precipitation and runoff separately.

In the second part, the parameters of a point variogram were estimated instead. For a certain point variogram, we estimated spatio-temporal variograms for the three catchment size classes by regularisation (Sect. 3.3). These regularised variograms were jointly compared to the sample variograms of the three catchment size classes. The same objective function was used as above, but the summation was over all catchment size classes. Regularised variogram models associated with the smallest objective function of ten trials are shown. The procedure was repeated for each variogram model and for precipitation and runoff separately. The parameters $\kappa$ and $\mu$ of Eq. (15) were also simultaneously fitted by this procedure, separately for each variogram model. The response time of the catchments is hence a result of the fitting procedure.

The scales of the diagrams of the spatio-temporal variograms are scaled linearly in terms of the bin spacing. As the bins have been selected approximately logarithmically (with the exception of zero lags) the axes are close to logarithmical.

\section{Results}

\subsection{Separately fitted variograms of precipitation}

The left column of Fig. 3 shows the spatio-temporal sample variograms of point and catchment precipitation, sorted by catchment size class. The total variance of precipitation is similar in time and space within the spatial and temporal extents of the data set $(300 \mathrm{~km}, 1000 \mathrm{~h}$ shown here). The variogram values increase with increasing spatial and tem- 


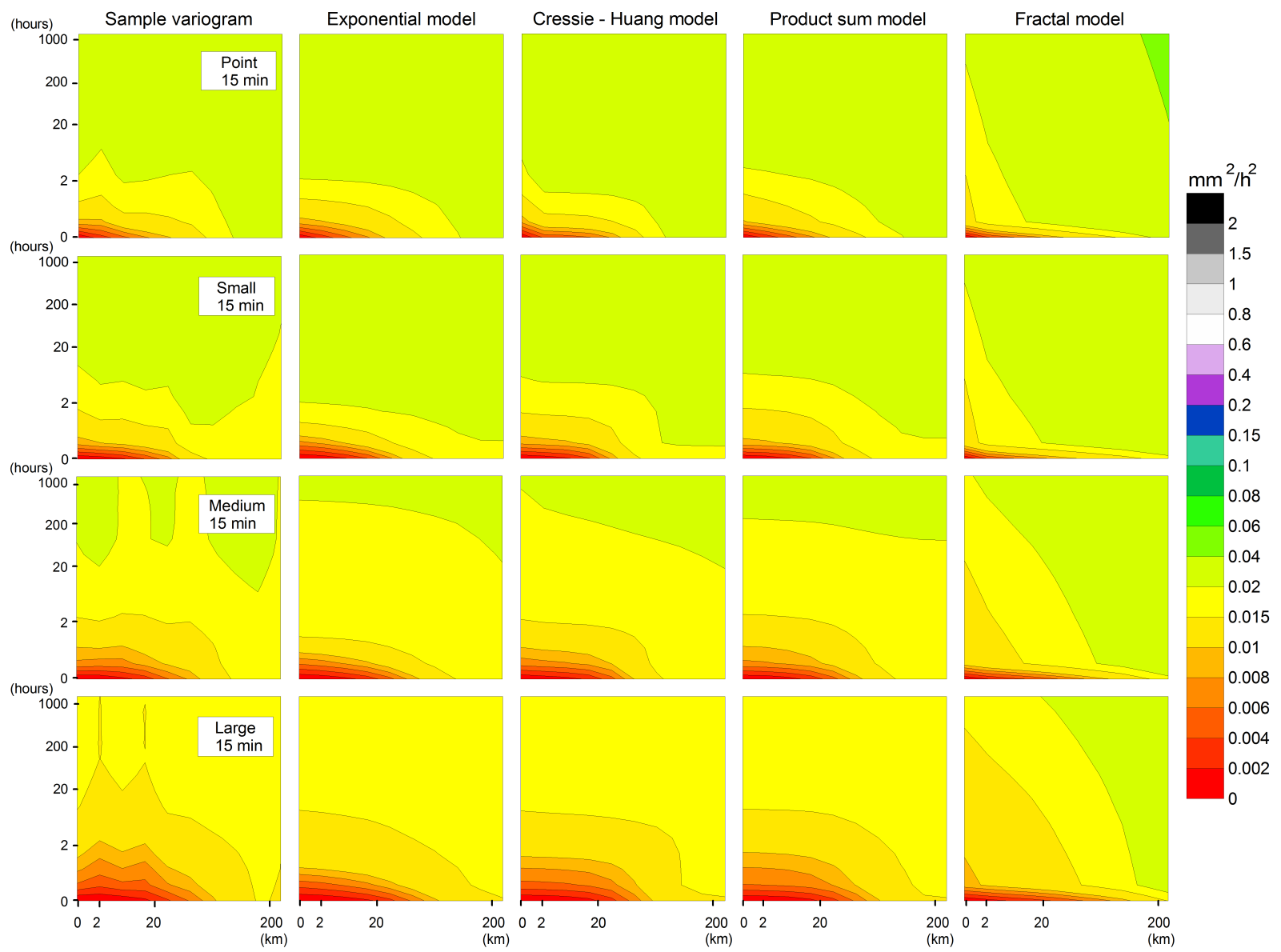

Fig. 3. Spatio-temporal variograms of catchment precipitation. Sample variograms (left column) and independently fitted variogram models (columns 2-5). The rows relate to different catchment size classes (small, medium, large) including point precipitation in the top row. The horizontal axes are space lag, the vertical axes are time lag.

poral distances which indicates the presence of spatial and temporal correlations as would be expected. There is a reduction in the variogram values as one moves from points to larger catchments which reflects the smoothing as a result of an increasing support. Columns two to five of Fig. 3 show the spatio-temporal variogram models that have been independently fitted to the sample variograms. For all models, with the exception of the fractal model, the visual fits are very good and the differences between the models are small. Fig. 4 shows the margins of the sample variograms and the fitted variogram models for precipitation. The margins of a spatio-temporal variogram are equivalent to the spatial and temporal variograms. The sample variograms are represented by points, while the fitted variograms are represented by lines. For a certain catchment size class, points and lines are of the same colour. All models, except for the fractal model, provide close fits. The shortest spatial lags show some differences between the models as this is where the models have been extrapolated beyond the data. Table 2 gives the values of the objective function for each variogram model and catchment size class as well as the average over the three size classes. We have also included the number of parameters to be fitted, including the two parameters of Eq. 15. The table indicates that the product-sum model can be best fitted to the sample variograms of precipitation. It should be noted that the product-sum model has the largest number of parameters, so the good fits may be both a result of a suitable model structure and the large number of degrees of freedom.

\subsection{Separately fitted variograms of runoff}

The left column of Fig. 5 shows the spatio-temporal sample variograms of runoff, sorted by catchment size class. The variograms indicate that there is a higher variance in space than there is in time within the spatial and temporal extents of the data set. The spatio-temporal variograms increase monotonously with spatial and temporal distances. There is a much stronger variance reduction effect between the variograms of the different catchment size classes than for precipitation. It is obvious that the catchment size has 

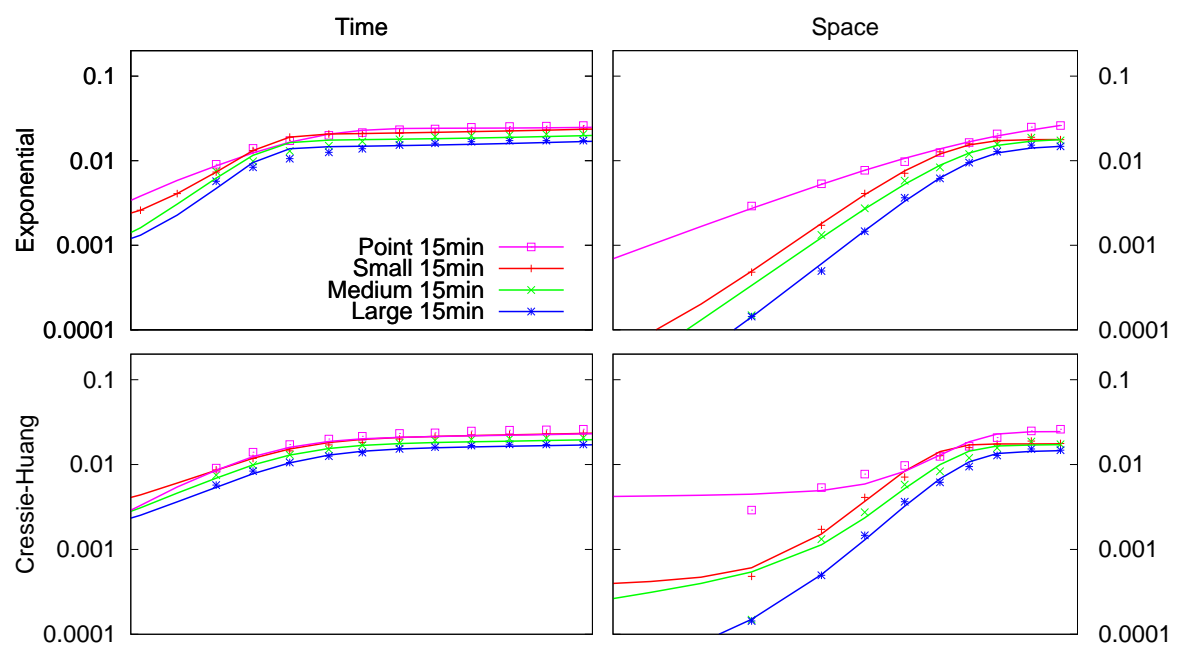

0.1
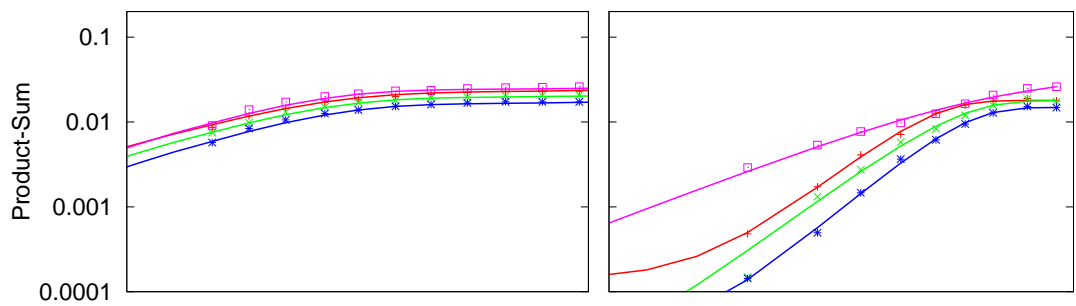

0.0001
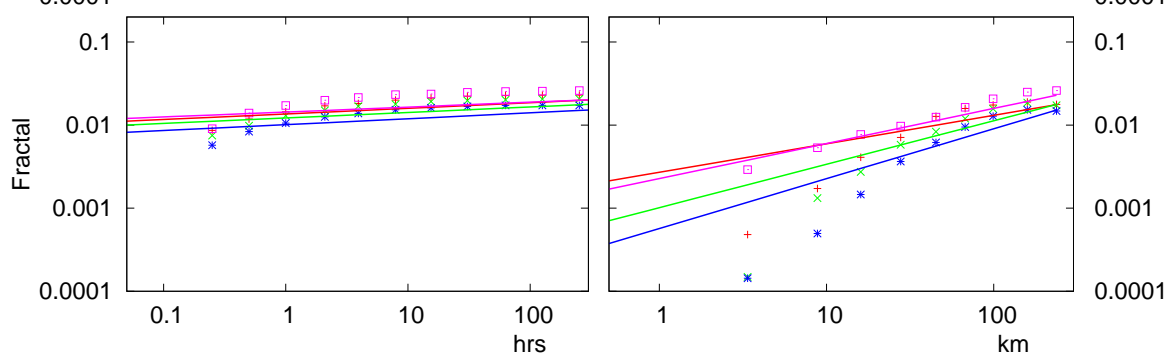

Fig. 4. Temporal (left) and spatial (right) margins of the spatio-temporal variograms of catchment precipitation as in Fig. 3 . Sample variograms are shown as points, fitted variogram models as lines.

Table 2. Objective function (Eq. 21) for the variogram models of precipitation fitted independently to spatio-temporal sample variograms for small, medium and large catchment size classes. Average refers to the average of the objective functions from the three catchment size classes. The number of parameters fitted consists of the parameters of the point variogram models and the two parameters of Eq. 15 .

\begin{tabular}{lcccccc}
\hline Variogram model & Point & $\begin{array}{c}\text { Small } \\
\text { catchments }\end{array}$ & $\begin{array}{c}\text { Medium } \\
\text { catchments }\end{array}$ & $\begin{array}{c}\text { Large } \\
\text { catchments }\end{array}$ & Average & $\begin{array}{c}\text { Number of } \\
\text { parameters }\end{array}$ \\
\hline Exponential model & 0.0036 & 0.0132 & 0.0122 & 0.0123 & 0.0126 & 10 \\
Cressie-Huang model & 0.0121 & 0.0122 & 0.0125 & 0.0062 & 0.0103 & 9 \\
Product-sum model & 0.0036 & 0.0094 & 0.0062 & 0.0042 & 0.0066 & 13 \\
Fractal model & 0.0992 & 0.1285 & 0.1310 & 0.1307 & 0.1301 & 6 \\
\hline
\end{tabular}

an efficient smoothing effect. Columns two to five of Fig. 5 show the variogram models that have been fitted separately for each catchment size class. All models can be fitted well to the sample variograms, with the exception of the fractal model, and the differences between the models are small. Figure 6 shows the margins of the sample variograms and the fitted variogram models. The figure shows in more detail the much stronger variance reduction from smaller to larger catchments than that of precipitation. Table 3 gives the values of the objective functions for the fitted variogram models. The product-sum model offers a slightly better fit than the exponential and the Cressie-Huang models. 


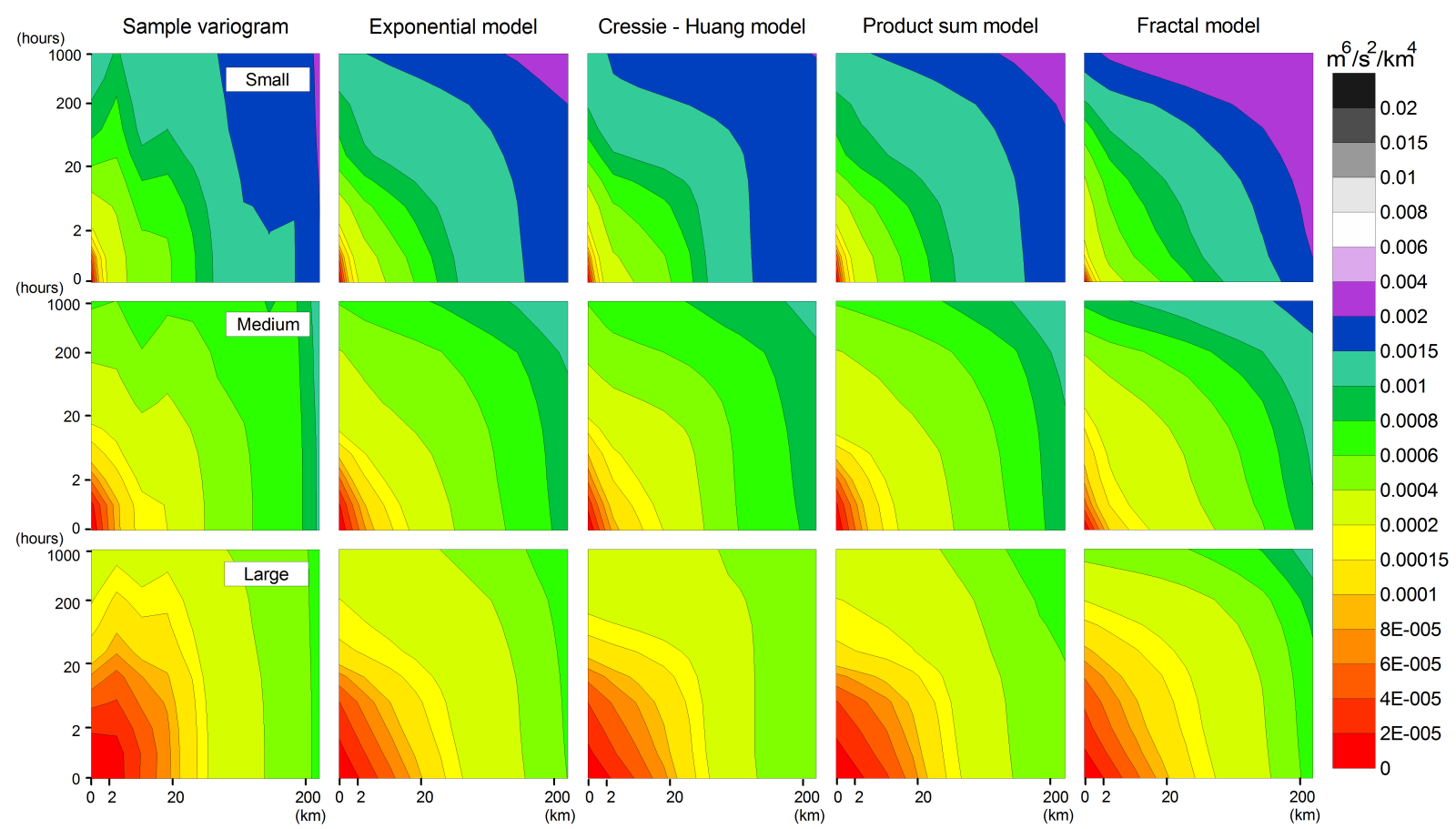

Fig. 5. Spatio-temporal variograms of runoff. Sample variograms (left column) and independently fitted variogram models (columns 2-5). The rows relate to different catchment size classes (small, medium, large). The horizontal axes are space lag, the vertical axes are time lag.

Table 3. Objective function (Eq. 21) for variogram models of runoff fitted independently to spatio-temporal sample variograms for small, medium and large catchment size classes. Average relates to the average of the objective functions for the three catchment size classes.

\begin{tabular}{lcccc}
\hline Variogram model & $\begin{array}{c}\text { Small } \\
\text { catchments }\end{array}$ & $\begin{array}{c}\text { Medium } \\
\text { catchments }\end{array}$ & $\begin{array}{c}\text { Large } \\
\text { catchments }\end{array}$ & Average \\
\hline Exponential model & 0.0094 & 0.0095 & 0.0151 & 0.0113 \\
Cressie-Huang model & 0.0135 & 0.0209 & 0.0216 & 0.0186 \\
Product-sum model & 0.0082 & 0.0083 & 0.0110 & 0.0092 \\
Fractal model & 0.0851 & 0.0878 & 0.0982 & 0.0904 \\
\hline
\end{tabular}

\subsection{Jointly fitted variograms of precipitation}

Figure 7 shows the results of jointly fitting the variograms of precipitation to the three catchment size classes. The variograms in the left column are again the sample variograms. The letters on the left side of the figure relate to the respective rows and denote estimation (E), verification (V) and fitting (F). The sample variograms of rows three, four and five have been used for the fitting of the models in columns two to five. With the exception of the fractal model, there are only small visual differences between the fitted variograms. The sample variogram of row two (point data of precipitation with a temporal support of $15 \mathrm{~min}$ ) can be used for verification. For this case, the differences between the models are slightly larger than for the fitting but the models are still rather close to the sample variogram, again with the exception of the frac- tal model. The top row of Fig. 7 shows the back-calculated point variograms valid for zero temporal and zero spatial supports, i.e. instantaneous point variograms. These do differ between the variogram models with the fractal and CressieHuang models giving larger sills than the other models. The exponential and product sum models are rather similar.

The margins of the variograms of Fig. 7 are shown in Fig. 8. The margins more clearly show that the overall fits are good to very good. The margins of the fractal model are less biased than the rest of the spatio-temporal variogram, especially along the spatial axis. As all catchment size classes have the same temporal support $(15 \mathrm{~min})$ the fractal model does not estimate any temporal variance reduction with increasing catchment size. The temporal variograms indicate that the Cressie-Huang and product-sum models slightly underestimate the temporal variance of point precipitation with 

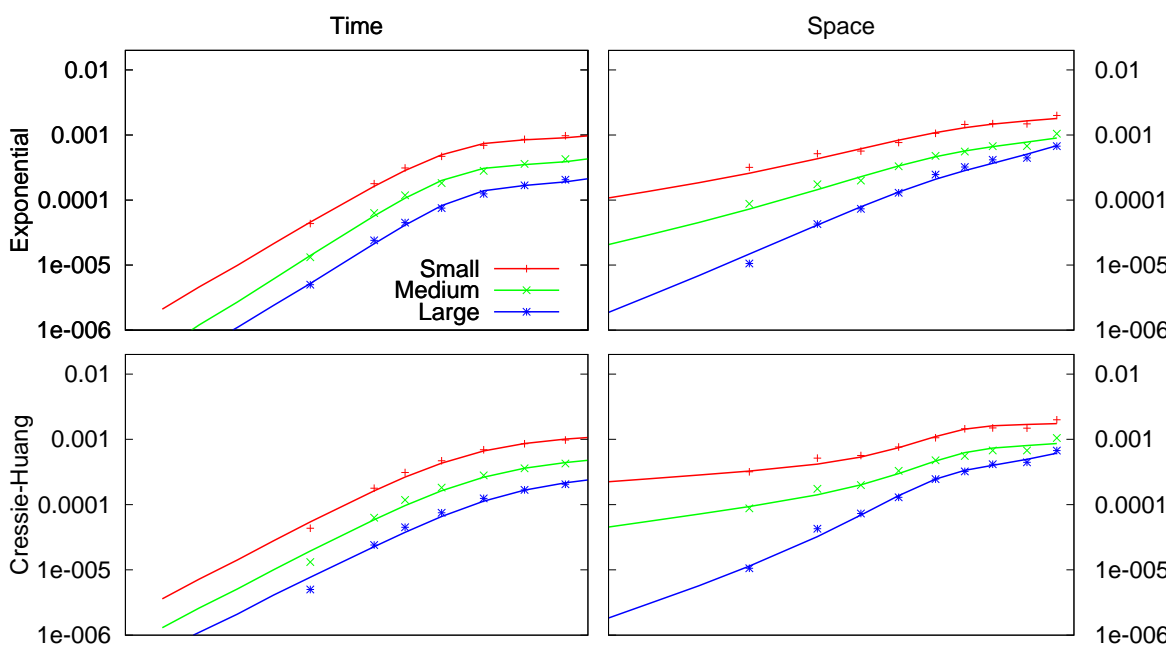

\subsection{1}

0.001

0.0001

$1 e-005$
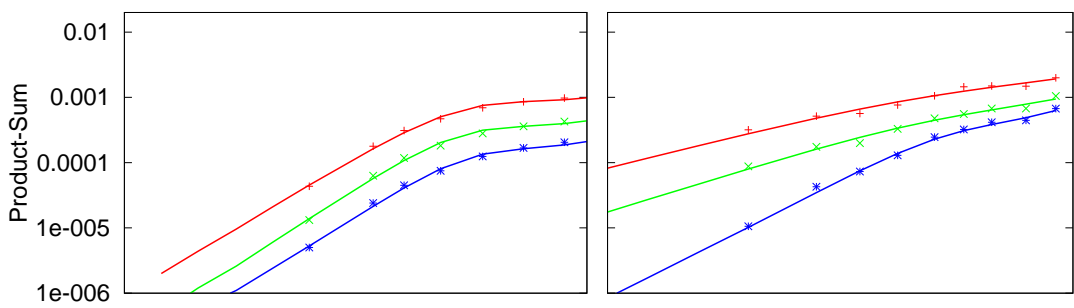

$1 \mathrm{e}-006$

0.01

0.001

0.0001

$1 \mathrm{e}-005$
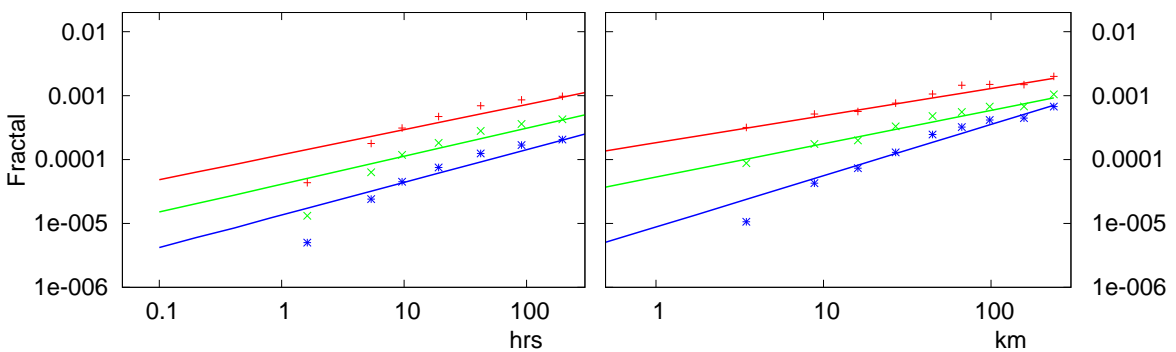

Fig. 6. Temporal (left) and spatial (right) margins of the spatio-temporal variograms of runoff as in Fig. 5. Sample variograms are shown as points, fitted variogram models as lines.

Table 4. Objective function (Eq. 21) for regularised variograms of precipitation fitted jointly to the spatio-temporal sample variograms for small, medium and large catchment size classes, denoted as "total". Point refers to the objective function for point precipitation with 15 min temporal support which is the verification case.

\begin{tabular}{lcc}
\hline Variogram model & Total (fitted) & Point (verification) \\
\hline Exponential model & 0.0113 & 0.0098 \\
Cressie-Huang model & 0.0145 & 0.0191 \\
Product-sum model & 0.0094 & 0.0304 \\
Fractal model & 0.1210 & 0.2549 \\
\hline
\end{tabular}

a temporal support of $15 \mathrm{~min}$. The exponential model performs best on the margins.

Table 4 shows the values of the objective function for the fitted variogram models. The product-sum model offers a slightly better fit than the exponential and the Cressie-Huang models but for the verification case (point precipitation with temporal support of $15 \mathrm{~min}$ ) the exponential model is the best model. The objective function for the goodness of fit (small, medium, large catchment sizes classes) is around 0.01 (with the exception of the fractal model) which is similar to the separate fitting (Table 2). This indicates that the regularisation is fully consistent with the catchment precipitation data. Note that the joint fitting (Table 4) has only one third of the free parameters of the separate fitting. This comparison tests the assumptions of regularisation in space, i.e., the approximation of the catchments by squares with an area equal to the median of each size class. It is clear that this approximation is sufficiently accurate for the purposes of regularisation. In the verification case (Table 4, right column) the errors are somewhat larger (0.01-0.03 depending on the model, excluding the fractal model) but in absolute terms this is still a small number. 


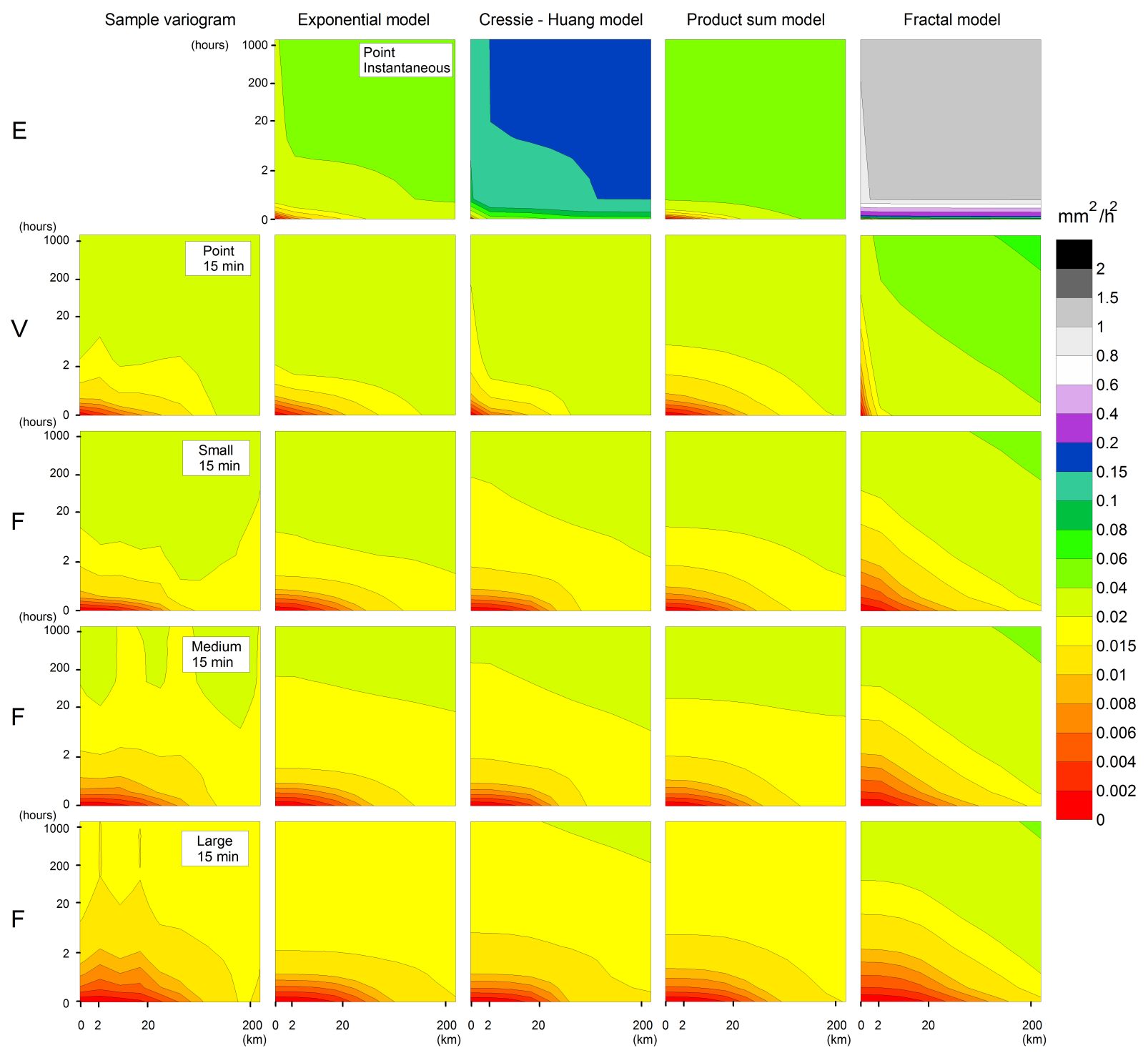

Fig. 7. Spatio-temporal variograms of catchment precipitation. Sample variograms (left column) and jointly fitted variogram models (columns 2-5, rows 3-5). Row 2 (point precipitation, temporal support of $15 \mathrm{~min}$ ) has not been used in the fitting and is used for verification. Top row shows the back-calculated variograms for zero spatial and temporal supports (instantaneous point precipitation). Letters "E", "V" and "F" stand for estimation, verification and fitting, respectively. The horizontal axes are space lag, the vertical axes are time lag.

\subsection{Jointly fitted variograms of runoff}

Regularised spatio-temporal variogram models were fitted to the sample variograms of runoff jointly for all catchment size classes and are shown in rows two to four of Fig. 9. There are only minor differences between the regularised variograms from the different models, and they are all similar to the sample variograms. The exception is the fractal model which cannot be fitted as well. It should be noted that this is the model with the smallest number of parameters, so a poorer fit would be expected. The point variogram models back-calculated by the procedure (Fig. 9, top row) exhibit significantly shorter spatial correlation lengths than any of the catchment scale variogram models. The point variogram models differ in terms of their sills (i.e. the overall levels). Similar to precipitation, the Cressie-Huang and fractal models have the largest sills. It is clear that there is substantial uncertainty associated with these variograms. However, for practical applications this may not be important if the spatiotemporal estimation of runoff is applied to catchments of a size range similar to that used here, as the regularised variograms based on these point variograms are all very similar.

Figure 10 shows the margins of the sample variograms and the fitted regularised models for runoff. There are only small differences between the exponential, Cressie-Huang and the product sum models. The temporal margins are al- 

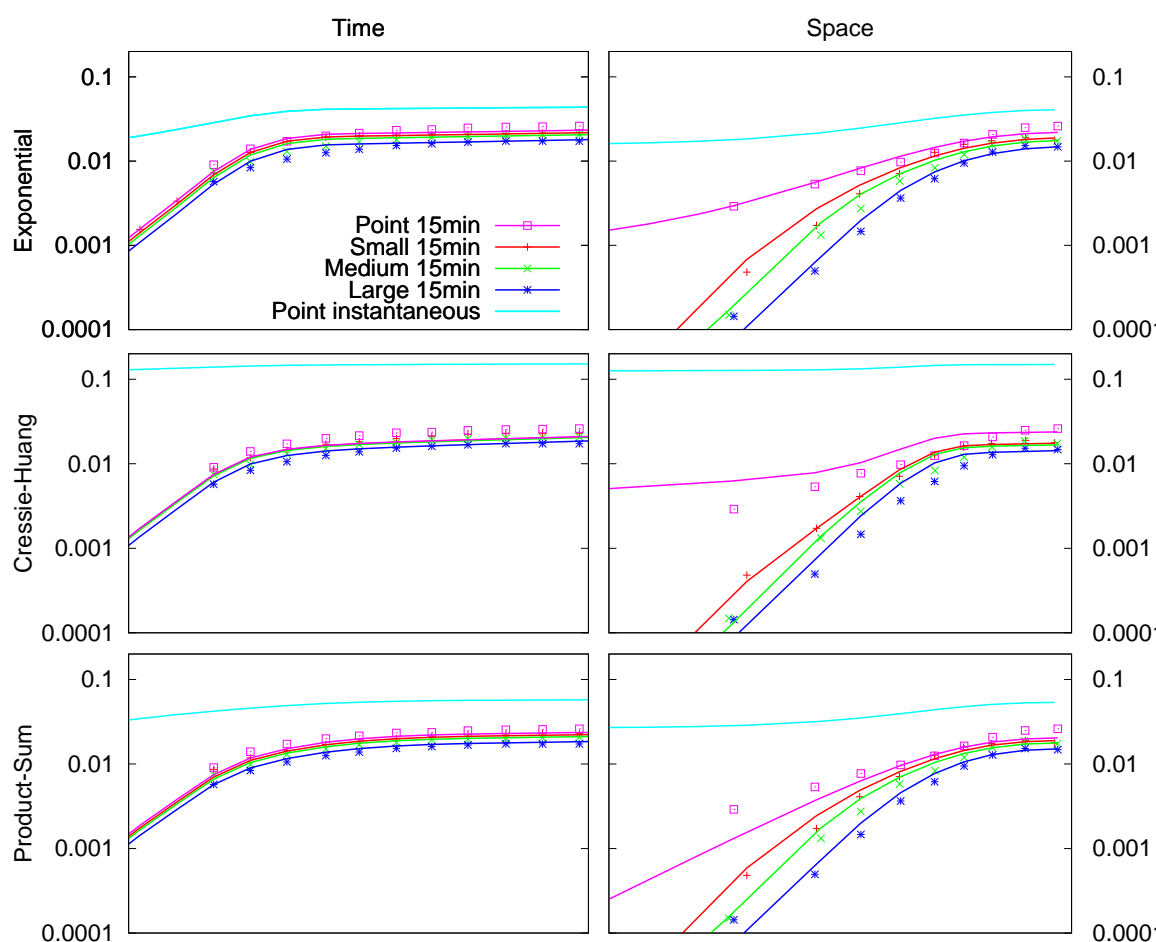

0.0001
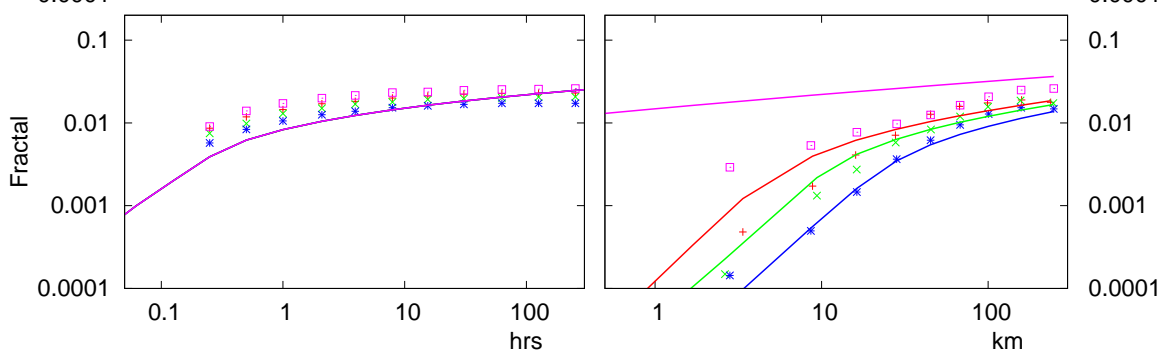

Fig. 8. Temporal (left) and spatial (right) margins of the spatio-temporal variograms of catchment precipitation as in Fig. 7 . Sample variograms are shown as points, jointly fitted variogram models (small, medium, large catchment size classes) as well as estimated variogram models (point $15 \mathrm{~min}$, point instantaneous) as lines.

Table 5. Objective function (Eq. 21) for regularised variograms of runoff fitted jointly to the spatio-temporal sample variograms for small, medium and large catchment size classes.

\begin{tabular}{lc}
\hline Variogram model & Total \\
\hline Exponential model & 0.0269 \\
Cressie-Huang model & 0.0227 \\
Product-sum model & 0.0257 \\
Fractal model & 0.1544 \\
\hline
\end{tabular}

most perfectly modelled, while there are minor deviations between the spatial sample variograms and the estimated variograms. The point variograms are shown in light blue. For the fractal model, the point variogram is larger than the range shown. Table 5 indicates that the variogram models give almost equally good fits with the exception of the fractal model. The objective functions of the exponential, CressieHuang and product sum models range between 0.02 and 0.03 . This is larger than those of the separately fitted variograms (around 0.01 in Table 3) which is likely related to the simplifications of the analysis including the assumptions on the unit hydrograph and the general assumption of linearity. However, the absolute values of the objective functions for the three models are still very small indicating overall excellent consistency.

All variogram models have been fitted ten times with different starting values which produced somewhat different parameter sets. This is because of local minima in the objective function. For the presentation we have selected the parameter sets with the smallest objective functions. To illustrate the uncertainty around these best fits we selected, for each model, the five best parameter sets and computed the average and the coefficient of variation (CV) for each parameter. 


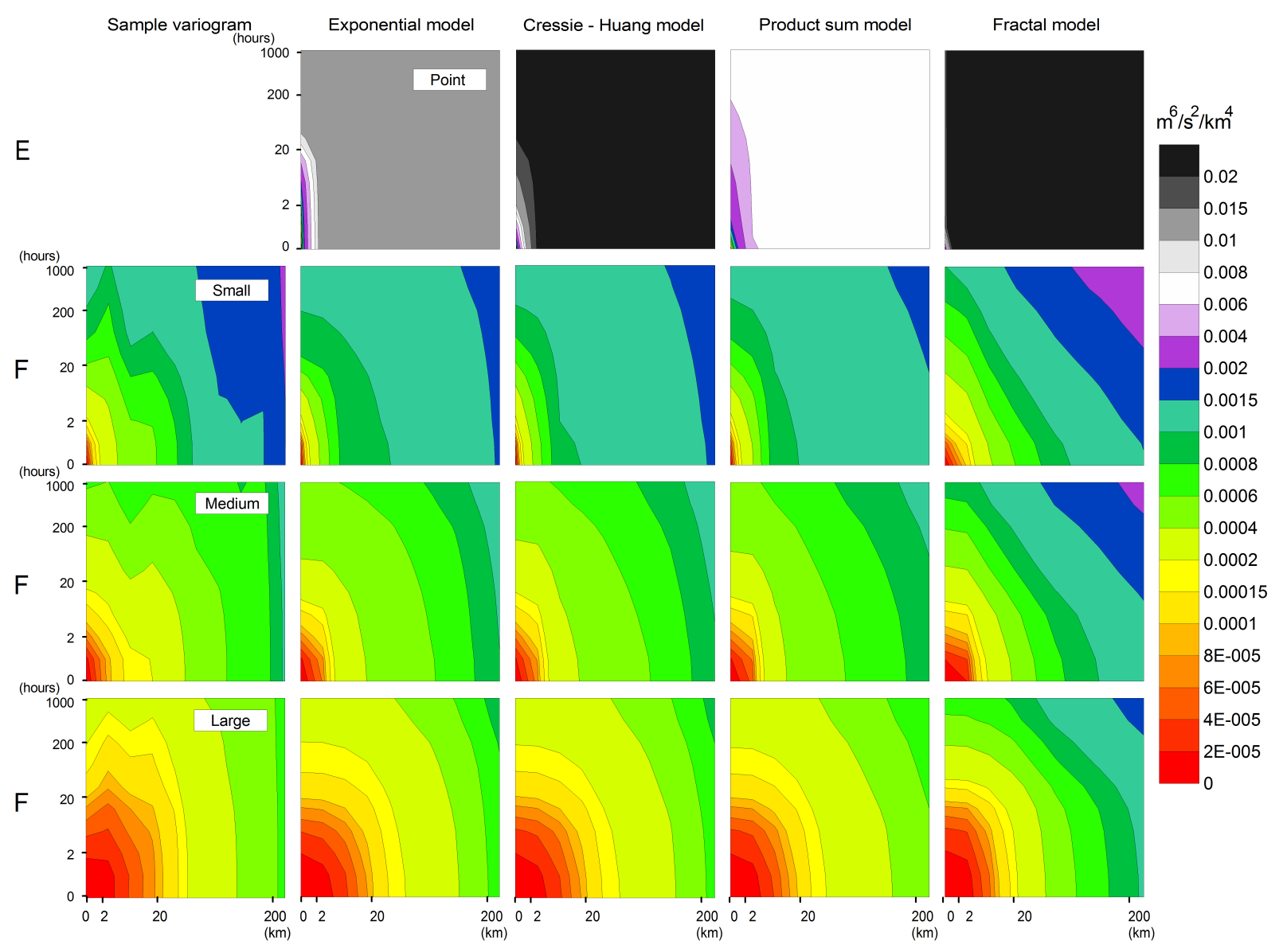

Fig. 9. Spatio-temporal variograms of runoff. Sample variograms (left column) and jointly fitted variogram models (columns 2-5, rows 2-4). Top row shows the back-calculated variograms for zero spatial and temporal supports (instantaneous point runoff ). Letters "E" and "F" stand for estimation and fitting, respectively. The horizontal axes are space lag, the vertical axes are time lag.

The CV is a measure of the uncertainty of the parameters. These statistics are shown in Table 6, together with the values of the corresponding objective functions. Overall, the uncertainty depends on the parameter estimated. For some parameters, the uncertainty is very small (e.g. $d_{1}$ ) but for other parameters the uncertainty is substantial. These differences are related to the sensitivity of the shape of the variogram to individual parameters. The parameters with the smallest sensitivity have the largest uncertainty but this may not be important for spatio-temporal estimation. It was more difficult to find suitable parameter sets for the product-sum model than for the other models. This is because it is the model with the largest number of parameters. In order to obtain suitable parameters, the parameter search was initiated with parameter sets found in previous optimisation runs. Because of the presence of local minima this tends to reduce the variability of the estimated parameters. The $\mathrm{CV}$ values of the parameters of the product-sum model in Table 6 hence tend to be smaller than those of the other models. A parameter that is of particular interest is the exponent in the relationship between space and time supports, $\kappa$. The uncertainty of this parameter ranges between 1 and 38\% depending on the model. The order of magnitude of $\kappa$ is hence a meaningful estimate. With the exception of the fractal model, for which the fitting was not very good, the $\kappa$ values of the different models are similar and range between 0.3-0.4. The parameters of the non-stationary parts $\left(a_{s}, a_{t}, \alpha\right.$ and $\left.\beta\right)$ are not well constrained as they are controlled by the large time scale and space scale variability present in the data. For the exponential and Cressie-Huang models, the levels (or sills) of the point variograms are defined by parameters $a_{1}$ and $a_{2}$, respectively. The $a_{2}$ value is significantly larger than $a_{1}$ reflecting the larger sills of the Cressie-Huang model as illustrated in Figs. 9 and 10.

\section{Conclusions}

\subsection{Sample variograms}

A comparison of the spatio-temporal variograms of runoff and precipitation indicates that, for a given catchment size 
Table 6. Statistics of the objective function $\left(\phi_{k}\right)$ of the joint fitting of the runoff variograms; estimated parameters of the space-time relationship of the supports $(\mu, \kappa)$; and estimated parameters of the point runoff variogram (remaining lines). $\mathrm{CV}$ is a measure of the uncertainty of the estimates.

\begin{tabular}{|c|c|c|c|c|c|c|c|c|}
\hline & \multicolumn{2}{|c|}{ Exponential model } & \multicolumn{2}{|c|}{ Cressie-Huang model } & \multicolumn{2}{|c|}{ Product-sum model } & \multicolumn{2}{|c|}{ Fractal model } \\
\hline & Average & $\mathrm{CV}$ & Average & $\mathrm{CV}$ & Average & $\mathrm{CV}$ & Average & $\mathrm{CV}$ \\
\hline$\phi_{k}$ & 0.0293 & 0.0786 & 0.0281 & 0.1176 & 0.0258 & 0.0158 & 0.1548 & 0.0020 \\
\hline$\mu$ & 1.8670 & 1.0422 & 2.5991 & 0.6058 & 2.0108 & 0.3027 & 0.3582 & 0.0710 \\
\hline$\kappa$ & 0.4193 & 0.3841 & 0.3440 & 0.2777 & 0.3065 & 0.1298 & 0.7936 & 0.0123 \\
\hline$a_{S}$ & 0.0000 & 1.2773 & 0.0007 & 0.9102 & 0.0000 & 0.1613 & 0.0840 & 0.5811 \\
\hline$a_{t}$ & 0.0024 & 1.4488 & 0.0005 & 1.0493 & 0.0001 & 0.4936 & 0.0277 & 0.6813 \\
\hline$\alpha$ & 0.5909 & 0.2939 & 0.2287 & 1.0096 & 0.6718 & 0.0385 & 0.0050 & 0.5056 \\
\hline$\beta$ & 0.1245 & 0.9893 & 0.0972 & 0.4798 & 0.1847 & 0.3471 & 0.0076 & 0.5847 \\
\hline$a_{1}$ & 0.0131 & 0.1046 & & & & & & \\
\hline$c_{1}$ & 0.0295 & 0.3589 & & & & & & \\
\hline$d_{1}$ & 1.0298 & 0.0198 & & & & & & \\
\hline$e_{1}$ & 1.6427 & 0.0548 & & & & & & \\
\hline$a_{2}$ & & & 0.0256 & 0.2456 & & & & \\
\hline$c_{2}$ & & & 0.1755 & 0.3966 & & & & \\
\hline$d_{2}$ & & & 1.2517 & 0.0976 & & & & \\
\hline$a_{3 s}$ & & & & & 0.0070 & 0.0773 & & \\
\hline$a_{3 t}$ & & & & & 0.0070 & 0.0798 & & \\
\hline$d_{3 s}$ & & & & & 1.6841 & 0.0805 & & \\
\hline$d_{3 t}$ & & & & & 31.6109 & 0.1490 & & \\
\hline$e_{3 s}$ & & & & & 1.6814 & 0.0090 & & \\
\hline$e_{3 t}$ & & & & & 0.5550 & 0.1742 & & \\
\hline$k$ & & & & & 142.8483 & 0.0780 & & \\
\hline
\end{tabular}

class, the variograms are fundamentally different. The left column of Fig. 5, as compared to the left column of Fig. 3, suggests that the plots of the runoff variograms are much more elongated in the time direction indicating that the time correlations of runoff are much more persistent than those of precipitation. Obviously, this is because of the time delays as rainfall passes through the catchment system. This is an effect of the catchment operating as a filter to the atmospheric forcing, with the time scale of the filter being directly related to the concentration time of the catchment. The contour lines of the variogram values give an indication of the characteristic velocities (Skøien et al., 2003). For precipitation of all catchment classes, a typical pair of length and times scales is $70 \mathrm{~km}$ and $2 \mathrm{~h}$ which suggests a typical characteristic velocity of $10 \mathrm{~m} / \mathrm{s}$. This is similar to the characteristic velocities found in Skøien et al. (2003) and consistent with the schematic of space time scales of Blöschl and Sivapalan (1995). For runoff, again for all catchment size classes, typical pairs of length and times scales are $2 \mathrm{~km}$ and $2 \mathrm{~h}, 20 \mathrm{~km}$ and $20 \mathrm{~h}$, and $50 \mathrm{~km}$ and $100 \mathrm{~h}$. This translates into typical characteristic velocities of $0.27,0.27$ and $0.14 \mathrm{~m} / \mathrm{s}$, respectively. These characteristic velocities are somewhat faster than those found in Skøien et al. (2003), which may be related to the higher temporal resolution of the data. The data resolve the event scale in more detail, hence one would expect the estimated scales to be associated with events. The slower characteristic velocities with increasing catchment size are likely related to the larger groundwater contribution in larger catchments.

Independently fitting variogram models to each catchment size class gave excellent to good fits for all the variogram models considered here with the exception of the fractal model. The product-sum model was generally better than the other models for both runoff and precipitation. The differences in the goodness of fit may be partly related to the degrees of freedom; the fractal model has the smallest number of parameters, the product-sum model the largest number of parameters. The objective function is dimensionless, so a comparison of precipitation and runoff is meaningful. The objective functions for runoff and precipitation are similar (both around 0.01 in Tables 2 and 3) indicating that the variogram models can be fitted equally well to runoff and precipitation.

The variograms change as one moves from small to medium sized and large catchments. The catchment scale effects are significantly larger for the case of runoff than for precipitation, i.e., in the case of runoff the variance reduction with catchment area is much larger (Figs. 3 and 5). Also, the temporal correlations increase more strongly with catchment area which, again, is related to the travel time of water in the 

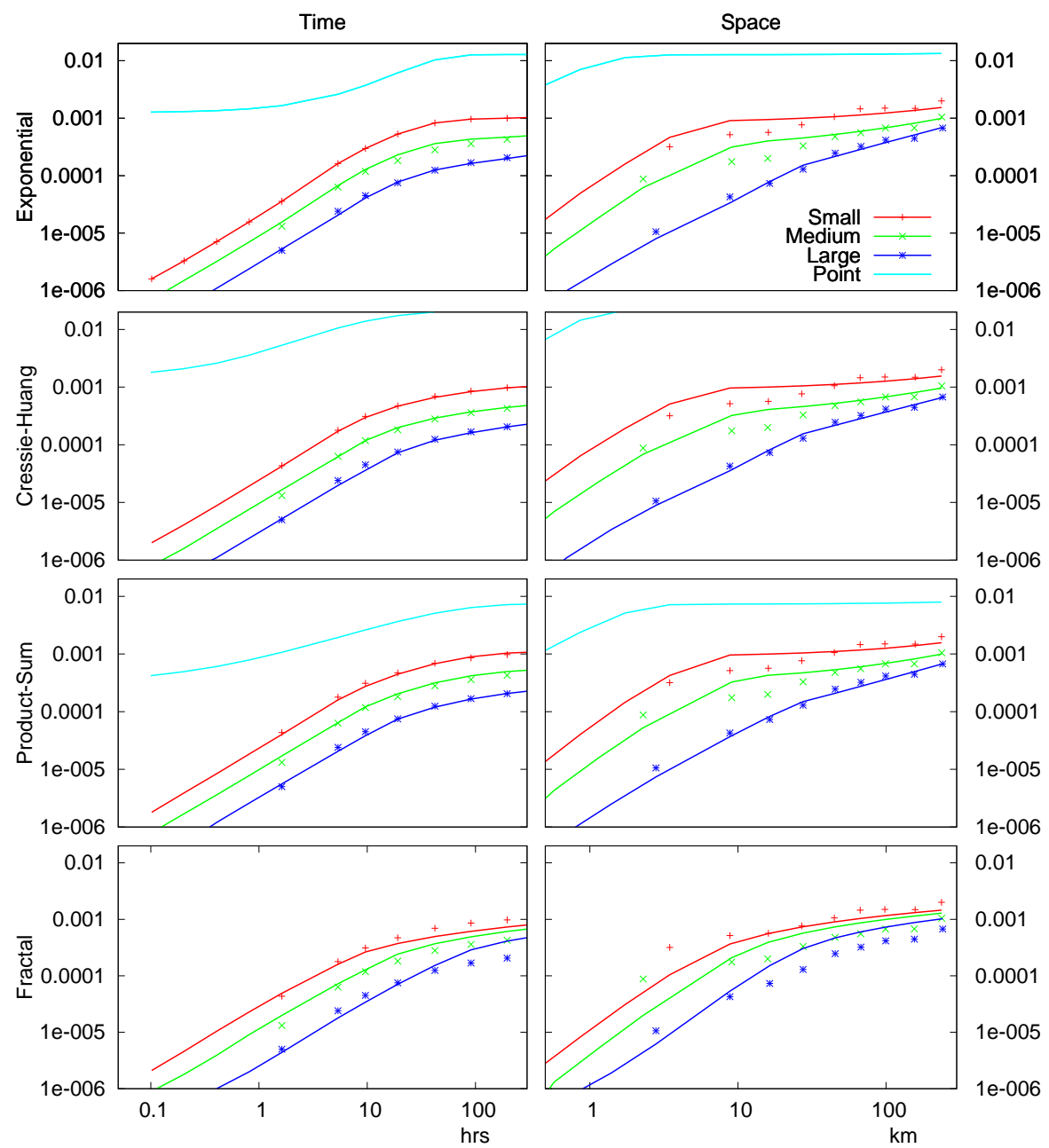

Fig. 10. Temporal (left) and spatial (right) margins of the spatio-temporal variograms of runoff as in Fig. 9. Sample variograms are shown as points, jointly fitted variogram models (small, medium, large catchment size classes) as well as estimated variogram model (point instantaneous) as lines.

catchments. The stronger time aggregation effects of runoff, as compared to precipitation, may explain the stronger variance reduction with spatial scale than that predicted by spatial aggregation, found by Woods et al. (1995).

\subsection{Space-time regularisation}

The regularisation of precipitation is used here for two purposes; to separate the spatial aggregation effects (moving from point rainfall to catchment rainfall) from spatiotemporal effects that involve runoff routing; and to test the spatial aggregation procedure, in particular the assumption of approximating catchments by squares and the use of a constant catchment size equal to the median in each size class. The comparison of back-calculated point precipitation (zero spatial support, 15 min temporal support) in Fig. 7, second row suggests that the assumptions are indeed appropriate for the data set used here, so the spatial regularisation of runoff, that uses the same procedure, is also valid. The objective functions of the joint fitting (Table 4) are close to the average objective functions obtained by the separate (direct) fitting (Table 2), i.e., around 0.01 in both cases (exponential, Cressie-Huang and product-sum models). This further corroborates the validity of the regularisation procedure.

For the case of runoff, however, the objective functions of the joint fitting (Table 5) are larger than those of the separate fitting (Table 3). For the joint fitting, the errors of the exponential, Cressie-Huang and product-sum models range between 0.02 and 0.03 , depending on the model, while they are around 0.01 for the separate fitting. This means that, for runoff, the space-time aggregation effects of catchments are not fully consistent with the assumptions made here. Specifically, the simplifications include the assumptions of a block unit hydrograph, the general assumption of linearity and, perhaps most importantly, a single relationship between catchment size and catchment response time. However, the overall 
magnitude of the objective functions are still very small (i.e., errors of 0.02-0.03) indicating that the first order effects of the spatio-temporal variability of runoff is indeed captured well by conceptualising catchments as linear space-time filters. The stronger time aggregation effects of runoff, as compared to precipitation, have been represented by a relationship between spatial and temporal supports (Eq. 15) which seems essential in representing the change of spatio-temporal runoff variograms with changing catchment size.

For precipitation, we found that the point scale productsum model provided slightly better variograms than the other models in terms of the goodness of fit to the small, medium and large catchment size classes but there was no advantage over the other models in the verification case of $15 \mathrm{~min}$ point precipitation. The fit of the fractal model was poorest but it was the model with the smallest number of parameters. There are two reasons for the poor fit of the fractal model. The obvious one is the smallest number of parameters among all variogram models, so the fractal model has the least flexibility. The other, probably equally important, reason is the lack of space-time interaction of the spatio-temporal fractal variogram, i.e., the fact that the partial derivatives of the variogram $\frac{\partial \gamma}{\partial h_{t}}$ and $\frac{\partial \gamma}{\partial h_{s}}$ only depend on $h_{t}$ and $h_{s}$, respectively. This lack of space-time interaction also concerns the fractal part of the other variograms, but to a lesser degree, as it only relates to a component of the entire variogram. For runoff, the goodness of fit of the exponential, Cressie-Huang and product-sum models was good (0.02-0.03) suggesting that all three models are suitable for the spatio-temporal estimation of runoff in the study area. Because of the small differences between the models, the choice of model could be based on computational convenience. It is interesting that the product-sum model reduces to a separable model with the fitted parameters, i.e. $a_{3 s}=a_{3 t}=0.0070$ and $k=142$, which is very close to the condition for the product-sum model to reduce to the separable model of Rodríguez-Iturbe and Mejía (1974). Separable models are computationally more convenient for some applications (Fuentes, 2006). The spatial variogram fits in this paper (Fig. 10 right column) are as good as or better than those of Skøien et al. (2003) (their Fig. 6b) who used spatial aggregation only. In addition, we can represent the temporal aggregation effects well (Fig. 10 left column).

\subsection{Interpretation of point variograms of runoff}

The point variograms of runoff, i.e. the variograms for a local runoff generation process with zero spatial and temporal supports, differ between the models. The fractal model gives the highest point variogram. This model, however, should be treated with caution as the model fits are not very good. For the Cressie-Huang model, the overall level or sill is higher than for the exponential and product sum models. The point model has been estimated from catchment size classes of 42 , 119 and $605 \mathrm{~km}^{2}$. These are the spatial supports. The associated temporal supports are, depending on the model (Eq. 15,
Table 6), approximately 7, 11 and $20 \mathrm{~h}$. This means that the back-calculation procedure involves substantial extrapolation to smaller scales, so the differences between the variogram models are not surprising. The shapes of the three point models are, however, not too different (Fig. 10 and Fig. 9 top row). It should also be noted that for estimation purposes one is usually interested in catchment sizes that are not much smaller than the smallest catchments considered here, e.g., $1 \mathrm{~km}^{2}$. For these catchment sizes, the variograms are much more similar. For the practical application of spatio-temporal estimation methods in catchment hydrology the differences in the point variograms may hence not be important.

The correlation lengths of the back-calculated point variograms of runoff are on the order of a kilometre or less, while the small catchments showed correlation lengths of around 10-20 km (Fig. 10 right column). Skøien et al. (2003) found a similar value of $0.7 \mathrm{~km}$ for point variograms of runoff. The short correlation lengths are plausible as local runoff will likely vary much over short spatial scales because of the variability of local infiltration and soil moisture characteristics (Western et al., 2002, 2004). It is also of interest to compare the sills or overall levels of the point precipitation and point runoff variograms. For the exponential, Cressie-Huang and product sum models of point/instantaneous precipitation the sills in space are $0.04,0.15$ and $0.06 \mathrm{~mm}^{2} \times \mathrm{h}^{-2}$, respectively (Fig. 8 right column). The corresponding values for runoff (Fig. 10 right column, with units adjusted) are 0.14, 0.26 and $0.12 \mathrm{~mm}^{2} \times \mathrm{h}^{-2}$, respectively. This means that the local variability of runoff is between twice and three times the variability of local rainfall. This is plausible as temporal and spatial soil moisture variability contributes to making local runoff more variable than rainfall. In time, local runoff is more coherent than rainfall (Fig. 10 left column as compared to Fig. 8 left column). This, again, is plausible because of the memory induced by soil moisture and local ponding. The non-stationary (fractal) parts of the variograms are more difficult to interpret. The parameters differ between the variogram models which is likely a result of the interdependence of the parameters of the fractal part and the other parameters of the variogram models. As the levels of the stationary parts of the point variograms differ, so will the non-stationary parts in the different models.

\subsection{Catchments as space-time filters}

The high temporal resolution of the data used here (15 min) allowed us to analyse the connections of space-time variability in more detail than has been possible in Skøien et al. (2003) who used daily data. A time step of $15 \mathrm{~min}$ resolves individual events even in the small catchment class. The kernel or space-time filter characteristics shed light on the space-time scaling behaviour of the rainfall-runoff transformation (Eq. 15). The parameter that is of particular interest is the exponent of the relationship between space and 
time supports, $\kappa$. With the exception of the fractal model, for which the fitting was not very good, the $\kappa$ values of the different models are similar and range between 0.3-0.4. With a $\mu$ value of around 2, this range of $\kappa$ gives response times of about $5 \mathrm{~h}$ and one day for catchments of 10 and $1000 \mathrm{~km}^{2}$ area, respectively. These are plausible event response times for the catchments considered here and are shorter than the low flow recession time scales to be expected in the catchments. The estimated response times seem to reflect large events with relatively short response times, as these events are associated with large absolute differences in the data set and hence contribute most to the second statistical moment. The estimated range of $\kappa$ represents the average scaling characteristics of catchments within the study region. Obviously, for an individual catchment, the response time may deviate significantly from the general relationship of Eq. (15). Figure 3 of Merz and Blöschl (2003), for example, shows a map of the deviations from such a general relationship based on an exponent of $\kappa=0.35$. However, interpreted as an ensemble average, the range found here is fully consistent with analyses of observed runoff response in numerous catchments (e.g. Fig. 11.4 of Anderson and Burt, 1990; Pilgrim 1987; Corradini et al., 1995). Similarly, typical values used in regional modelling studies are within this range. For example, Blöschl and Sivapalan (1997) used an exponent of $\kappa=0.35$ in analysing the spatial scaling behaviour of flood frequency. The consistency suggests that the concept of catchments as space-time filters is indeed meaningful. It should be noted that, if known, the deviations of catchment response times from a general relationship could be included in the spacetime filter framework proposed here.

Spatio-temporal point variograms of runoff as derived in this paper could be used in spatio-temporal estimation models similar to the TOPKRIGING approach of Skøien et al. (2005). Initial analyses (Skøien and Blöschl, 2005) have demonstrated the feasibility of such an approach for estimating runoff time series in ungauged catchments. This method would be particularly useful for filling in missing data of streamflow records based on the records in neighbouring catchments. Clearly, the approach would be expected to work best if the density of the stream network is high as is the case in Austria. If fewer stream gauges are available the variograms will not be as well defined (see Skøien and Blöschl (2006a, b) for sampling issues) while knowledge about the physical characteristics of the catchment and climate systems will become relatively more important. We have treated the precipitation variograms separately from the runoff variograms in this paper. Another extension of the work reported here would be to combine these two analyses. This could be based on similar concepts as those proposed by Woods and Sivapalan (1999).

Acknowledgements. Our research work has been supported financially by the Austrian Academy of Sciences project HOE18. We would like to thank the Hydrographic Office at the Federal Ministry of Agriculture, Forestry, Environment and Water Management in Austria for providing the Austrian data. We would also like to thank the two reviewers Alberto Montanari and Lars Gottschalk for their valuable comments.

Edited by: G. Pegram

\section{References}

Anderson, M. G. and Burt, T. P.: Subsurface runoff, in: Process Studies in Hillslope Hydrology, edited by: Anderson, M. G. and Burt, T. P., Wiley, Chichester, 365-400, 1990.

Blöschl, G. and Sivapalan, M.: Process controls on regional flood frequency: Coefficient of variation and basin scale, Water Resour. Res., 33, 2967-2980, 1997.

Blöschl, G. and Sivapalan, M.: Scale issues in hydrological modelling - a review, Hydrol. Processes, 9, 251-290, 1995.

Bochner, S.: Harmonic Analysis and the Theory of Probability, University of California Press, Berkeley and Los Angeles, 176 pp., 1955.

Corradini, C., Melone, F., and Singh, V. P.: Some remarks on the use of GIUH in the hydrologic practice, Nordic Hydrol., 26, 297$312,1995$.

Cressie, N. and Huang, H. C.: Classes of nonseparable, spatiotemporal stationary covariance functions, J. Amer. Stat. Assoc., 94, 1330-1340, 1999.

Cressie, N.: Fitting variogram models by weighted least squares, Math. Geol., 17, 563-586, 1985.

Cressie, N.: Statistics for spatial data, Wiley, New York, NY, 1991.

De Cesare, L., Myers, D. E., and Posa, D.: Estimating and modeling space-time correlation structures, Statistics \& Probability Letters, 51, 9-14, 2001.

De Iaco, S., Myers, D. E., and Posa, D.: Space-time analysis using a general product-sum model, Statistics \& Probability Letters, 52, 21-28, 2001.

Duan, Q., Sorooshian, S., and Gupta, V. K.: Effective and Efficient Global Optimization for Conceptual Rainfall-runoff Models, Water Resour. Res., 28, 1015-1031, 1992.

Fuentes M.: Testing for separability of spatial-temporal covariance functions, J. Stat. Planning and Inference, 136, 447-466, 2006.

Gottschalk, L.: Correlation and covariance of runoff, Stochastic hydrology and hydraulics, 7, 85-101, 1993a.

Gottschalk, L.: Interpolation of runoff applying objective methods, Stochastic hydrology and hydraulics, 7, 269-281, 1993b.

James, B. R. and Freeze, R. A.: The worth of data in predicting aquitard continuity in hydrogeological design, Water Resour. Res., 29, 2049-2065, 1993.

Journel, A. G. and Huijbregts, C. J.: Mining geostatistics, Academic Press, London, UK, 600 pp., 1978.

Kyriakidis, P. C. and Journel A. G.: Geostatistical space-time models: a review, Math. Geology, 31, 651-684, 1999.

Merz, R. and Blöschl, G.: A process typology of regional floods, Water Resour. Res., 39(12), SWC51-SWC520, doi:10.1029/2002WR001952, 2003.

Merz, R., Blöschl, G., and Parajka, D.: Raum-zeitliche Variabilität von Ereignisabflussbeiwerten in Österreich (Spatial-temporal variability of event runoff coefficients in Austria), Hydrologie und Wasserbewirtschaftung, 50, 2-11, 2006. 
Mitchell M. W., Genton, M. G., and Gumpertz M. L. Testing for separability of space-time covariances, Environmetrics, 16, 819831, 2005.

Pilgrim, D. H. (Ed.): Australian Rainfall and Runoff, The Institution of Engineers, Barton, ACT, Australia, 374 pp., 1987.

Renard, P., Demougeot-Renard, H., and Froidevaux, R. (Eds.): Geostatistics for Environmental Applications, Springer, Berlin Heidelberg, New York, 480, 2005.

Rodríguez-Iturbe, I. and Mejía, J. M.: The Design of rainfall networks in time and space, Water Resour. Res., 10, 713-728, 1974.

Sauquet, E., Gottschalk, L., and Leblois, E.: Mapping average annual runoff: a hierarchical approach applying a stochastic interpolation scheme, Hydrol. Sci. J., 45, 799-815, 2000.

Sivapalan, M., Takeuchi, K., Franks, S. W., Gupta, V. K., Karambiri, H., Lakshmi, V., Liang, X., McDonnell, J. J., Mendiondo, E. M., O’Connell, P. E., Oki, T., Pomeroy, J. W., Schertzer, D., Uhlenbrook, S., and Zehe, E.: IAHS Decade on Predictions in Ungauged Basins (PUB), 2003-2012: Shaping an exciting future for the hydrological sciences, Hydrol. Sci. J., 48, 857-880, 2003.

Skøien, J. O. and Blöschl, G.: Sampling scale effects in two dimensional random fields and implications for environmental modelling, Environ. Monit. Assessment 114, 521-552, 2006a.

Skøien, J. O. and Blöschl, G.: Scale effects in estimating the variogram and implications for soil hydrology, Vadose Zone J 5, 153-167, doi:10.2136/vzj2005.0069, 2006b.
Skøien, J. O., Blöschl, G., and Western, A. W.: Characteristic space-time scales in hydrology, Water Resour. Res., 39(10), SWC111-SWC1119, doi:10.1029/2002WR001736, 2003.

Skøien, J., Merz, R., and Blöschl, G.: Top-kriging - geostatistics on stream networks, Hydrol. Earth Syst. Sci., 10, 277-287, 2005, http://www.hydrol-earth-syst-sci.net/10/277/2005/.

Skøien, J. O. and Blöschl, G.: Geostatistical interpolation of runoff, EGU General Assembly 2005, Vienna, Austria, 24-29 April 2005, Geophysical Research Abstracts, 7, EGU05-A-06526, 2005.

Taylor, G. I.: The spectrum of turbulence, Proc. R. Soc. Lond. A., 164, 476-490, 1938.

Western, A. W. and Blöschl, G.: On the spatial scaling of soil moisture, J. Hydrol., 217, 203-224, 1999.

Western, A. W., Zhou, S.-L., Grayson, R. B., McMahon, T. A., Blöschl, G., and Wilson, D. J.: Spatial correlation of soil moisture in small catchments and its relationship to dominant spatial hydrological processes, J. Hydrol., 286(1-4), 113-134, 2004.

Western, A., Grayson, R., and Blöschl, G.: Scaling of soil moisture: a hydrologic perspective, Ann. Rev. Earth Planet. Sci., 30, 149180, 2002.

Woods, R. and Sivapalan, M.: A synthesis of space-time variability in storm response: Rainfall, runoff generation, and routing, Water Resour. Res., 35, 2469-2485, 1999.

Woods, R., Sivapalan, M., and Duncan, M.: Investigating the representative elementary area concept: An approach based on field data., Hydrol. Process., 9, 291-312, 1995. 